\title{
Proposed WiMAX Hybrid Scheduler with Split FTP Traffic and its Performance Evaluation
}

\author{
Anju Lata Yadava*, P. D. Vyavahare ${ }^{\mathrm{b}}$, P. P. Bansod ${ }^{\mathrm{c}}$ \\ ${ }^{a, b, c}$ Shri G.S. Institute of Technology and Science, 23 Sir M. Visvesvaraya Marg,Indore (M.P), India
}

Received: 03 November 2017; Accepted: 13 February 2018; Published: 08 November 2018

\begin{abstract}
The most promising broadband wireless access technology, Worldwide interoperability for Microwave Access (WiMAX), is the commercial name of IEEE 802.16. It is popular due to its middle range access mobile, high data rates, high scalability and convenient deployment. WiMAX provides Quality of Service (QoS) to various triple-play services (voice, video and data) through five service classes. QoS provisioning to various service classes in WiMAX is achieved by a scheduler at MAC layer. Selection of appropriate scheduler is based on certain design goals, such as attainment of QoS required for variety of applications, fairness to all services, reduced complexity etc. However, in general, File Transfer Protocol (FTP) traffic starves for bandwidth under the presence of high priority real-time traffic.

This paper proposes a WiMAX hybrid scheduler that integrates homogeneous schedulers namely Weighted Fair Queuing (WFQ), strict priority and a Round Robin (RR). The unique feature of the proposed scheduler is that it has a splitter for FTP traffic with varying ratio and two stage priority schedulers for providing proper traffic load distribution for packet scheduling. The analytical modelling of proposed scheduler is achieved by Markov Chain and balance equations are solved to derive performance indicators such as mean queue length of packets, mean queuing delay, throughput and inter-class fairness for various triple-play services. The analytical results show that, as compared to recently reported hybrid WiMAX schedulers, proposed hybrid scheduler fulfils QoS requirements of various services and the splitting of FTP traffic has improved fairness among various services.
\end{abstract}

Index Terms: Hybrid scheduling algorithms in WiMAX; Quality of Service (QoS); Markov chain; Analytical Model; Performance Evaluation of WiMAX.

(C) 2018 Published by MECS Publisher. Selection and/or peer review under responsibility of the Research Association of Modern Education and Computer Science

\footnotetext{
* Corresponding author. Tel: 9425437959

E-mail address: yadawanjulata@gmail.com
} 


\section{Introduction}

In wireless networks there has been an increase in heterogeneous traffic each having different QoS requirements. The WiMAX network provides a feasible option for last mile Internet access. WiMAX is a Wireless Metropolitan Area Networks (WMAN) standard that operates at 2-11 GHz frequency range for Non Line Of Sight (NLOS) and 10-66 GHz for Line Of Sight (LOS) transmission. Theoretically, WiMAX Base Station (BS) can provide wireless access coverage upto $50 \mathrm{Km}$ for fixed Subscriber Station (SS) and upto 10 $\mathrm{Km}$ for Mobile Station (MS) with maximum of data rate upto 70 Mbps [1]. WiMAX support for QoS is achieved at the Medium Access Control (MAC) layer by differentiation of traffic types through five service classes: Unsolicited Grant Services (UGS), extended real-time Polling Service (ertPS), real-time Polling Service (rtPS), non-real-time Polling Service (nrtPS) and Best Effort (BE). UGS class of service has fixed bandwidth allocation while a particular scheduling policy distributes remaining bandwidth to ertPS, rtPS, nrtPS and BE.

IEEE 802.16 mentions abstraction of QoS requirements for different service classes but does not describe the techniques to be used to achieve QoS. Thus, it is an open area of exploration for researchers and service providers. Packet scheduling plays a crucial role in QoS provisioning in broadband wireless networks. Schedulers provide mechanisms for bandwidth allocation and multiplexing of packets. In WiMAX, scheduler is implemented in BS to allocate slots to uplink and downlink connections [2]. However, the scheduling mechanism has not been defined by the standard. The QoS requirements vary across applications, services, and users. A voice transmission system has low data rate requirements but the total delay must be less than $200 \mathrm{~ms}$, otherwise the delay is intolerable for the user. On the other hand, a data system requires much higher data rates and a very small Bit Error Rate (BER). Real-time video applications require high data rate along with the low delay latency constraints similar to voice system.

WiMAX schedulers can be principally grouped into three types: homogeneous, hybrid and opportunistic. Homogeneous schedulers are legacy scheduling algorithms. Such schedulers do not take into account the nature of channel errors or packet loss rates while deciding scheduling policies [3]. They usually serve as intra-class schedulers. Hybrid scheduler aggregates more than one homogenous schedulers. Opportunistic scheduler considers the channel fluctuations for achieving higher throughput by providing higher scheduling priority to the SSs with better channel Signal to Noise Ratio (SNR) as compared to SSs with poor channel SNR [4]. However, such schedulers require lot of real-time information and are complex in implementation. Therefore, devising hybrid WiMAX scheduler is an active area of research.

In general, FTP and data traffic accounts for the major portion of total traffic. Therefore, there is a need to handle these traffics in such a way that their requirement of high throughput is satisfied and they are not starved under the presence of high priority real-time traffic. The paper proposes and analyzes an improved hybrid scheduler with FTP traffic splitter and two priority schedulers to provide QoS to various applications such as voice, video, and data including FTP. The Markovian model is utilized to analyze the proposed hybrid scheduler model. Balance equations are realized from the analytical model and solved using matrix multiplication approach to derive expressions for performance indicators such as mean queue length of packets, mean queuing delay, throughput and inter-class fairness.

The paper is organized as follows: Section II includes the related literature survey. In Section III, the architecture of proposed hybrid scheduler with split traffic is described in detail. The analytical model of the proposed hybrid scheduler is described in Section IV. In Section V, the proposed hybrid scheduler is analyzed based on various performance indicators such as mean queue length of packets, mean queuing delay and throughput for voice, video, FTP and data traffic and inter-class fairness for different queue buffer size configurations. Finally, the paper is concluded in Section VI. 


\section{Related Works}

Scheduling of packets is the technique of transmission queue management for packets originating from various services, while dealing with problem of shared bandwidth in a wireless medium. The scheduling scheme fulfils the requisite QoS parameters (delay, throughput) of each service to readily exploit the uplink traffic bandwidth. In WiMAX, BS scheduler implements a policy to determine total number of slots to be assigned to each SS. Effective scheduler balances the requisite QoS parameters as required by each application and each user with the accessible radio bandwidth. IEEE 802.16 does not define actual packet scheduling mechanism for providing QoS in MAC layer and it is left open to the researchers and system implementers [5].

The arrival of packets at BS is followed by their grouping into different connections and various service flows. Separate queues are maintained for different types of traffic. Packets are executed in first come first served basis within a queue. Various scheduling schemes such as Round Robin (RR), Weighted Round Robin (WRR) or Earliest Deadline First (EDF) operate according to the function implemented to fulfil requisite QoS parameters of various services [6]. In wireless environment, a scheduler may provide an efficient operation by utilizing the location dependent and time dependent channel state during decision phase. In such cases, a channel monitor is required to examine the channel state for each station. Some schedulers consider various parameters such as: 'traffic service type, set of QoS requirements of the connections, available bandwidth for transmission, bandwidth requirements from the connections and waiting time of bandwidth request in the system' as a part of scheduling strategy [7].

The performance of communication networks under fluctuating traffic is widely modeled using Markov chain. Various WiMAX hybrid schedulers have been recently reported in literatures and various investigations have directed towards analytical models of hybrid schedulers. A hybrid scheduler which integrates Priority Queue (PQ) and Weighted Fair Queuing (WFQ) schedulers is proposed in [8]. Non-bursty Poisson process and bursty Markov Modulated Poisson Process (MMPP) characterizes arrival process of traffic. The model assigns highest priority to voice traffic and the WFQ scheduler schedules video and FTP traffic. The model satisfies QoS requirements of only three service classes with a lower inter-class fairness. The hybrid scheduler proposed in [9] schedules voice, video, FTP and data traffic and is analytically modeled using Markov Chain. The voice and video traffic (served by a WFQ scheduler) are assigned higher priority than FTP and data traffic (served by a RR scheduler). The model does not satisfy QoS requirements of all applications, though it provides high inter-class fairness. In [10], a queue decomposition technique was schemed that represented the Priority Queuing (PQ) system by a combination of a group of Single-Server Single-Queue (SSSQ) to realize their service capacities. Markov chain models the hybrid scheduler and stationary probabilities are retrieved using closed loop expressions and matrix geometric method.

An uplink and downlink scheduler is laid out by [11], in which resources are calculated and allotted in terms of number of slots, depending on the requisite QoS parameters and priority of service classes. The scheduler satisfies delay and throughput needs of all applications in WiMAX. However, the service classes are scheduled by strict priority which can cause the starvation of lower priority service classes. A two state MMPP process is used to model the arrival of VoIP packets for a WiMAX network in [12]. Analytically the performance parameters are obtained and compared with system in which single queue is used to buffer VoIP packets and system with active queue management. For scheduling of rtPS, nrtPS and BE class, authors in [13] proposed an analytical model of two level scheduling, based on Markov chain. The proposed scheme consists of a call admission control and a packet scheduler including the inter-class scheduler and intra-class scheduler. In [14], a scheduler based on queue length has been proposed to guarantee required minimum throughput to nrtPS class. The proposed algorithm outperforms RR scheduler. A computational scheme, Passage Relocation Admission Control (PRAC) protocol, which is an extended version of downlink scheduling approach, has been proposed by [15]. An analytical model based on Markov chain has been assessed to evaluate network performance. The model provides an improved performance as compared with the existing fuzzy logic-based scheduling scheme. 


\section{Proposed WiMAX Hybrid Scheduler with Split FTP Traffic}

The main idea behind WiMAX scheduler's proposal is to satisfy QoS requirements of packets originating from both real-time and non-real-time traffic. The proposed hybrid scheduler consists of WFQ, RR, a splitter and strict priority queues and their interconnections so as to combine the advantages of different scheduler strategies for various types of traffic in a communication network [16]. It is implemented at SS in a WiMAX network for uplink scheduling.

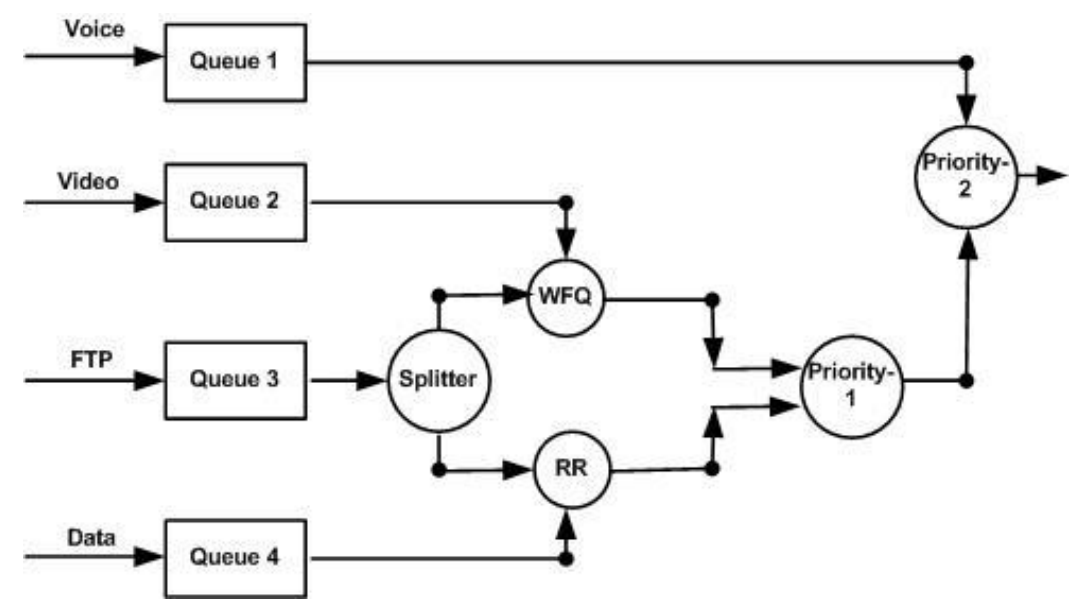

Fig.1. Architecture of proposed Hybrid Scheduler with Split FTP Traffic (HS-ST)

The proposed hybrid scheduler with split FTP traffic (as shown in Fig. (1)), consists of four queues $\mathrm{Q}_{1}, \mathrm{Q}_{2}$, $\mathrm{Q}_{3}$ and $\mathrm{Q}_{4}$ to cache packets of voice, video, FTP and data traffic, respectively. The voice traffic, being delay intolerant, is given highest priority. The FTP traffic undergoes a splitting process before being queued in WFQ or RR queue with variations in split ratio as one of the design parameter. Traffic splitter is included in the model to provide load distribution of FTP packets, as FTP traffic accounts for large volume of traffic. It is required that FTP traffic should have high throughput and does not undergo starvation of bandwidth due to high priority real-time traffic. The video traffic and the first portion of split FTP traffic are scheduled by WFQ. The data traffic and the second part of split FTP traffic are scheduled using RR scheduler. Finally, two priority schedulers combine the traffic to schedule a packet transmission. A priority scheduler always schedules the packet with higher priority first. Only in the absence of high priority packets, the low priority packets are scheduled.

\section{Analytical Modeling of Proposed WiMAX Hybrid Scheduler with Split FTP traffic}

The proposed WiMAX hybrid scheduler with split FTP traffic is analyzed by a model having single server and multiple queues, one queue meant for each service class. The scheme is modelled as $\mathrm{M} / \mathrm{M} / 1 / \mathrm{K}$ queuing system with four queues, each of maximum size $\mathrm{K}_{1}, \mathrm{~K}_{2}, \mathrm{~K}_{3}$ and $\mathrm{K}_{4}$. To simplify the analysis, certain assumptions are made. The arrival of packets are assumed to be Poisson distributed. The incoming traffic arrival rate for class c (c $\varepsilon$ (ertPS, rtPS, nrtPS or BE)) is $\lambda_{\mathrm{c}}$ (packets/sec). In the analytical equations ertPS corresponds to $\mathrm{c}=1$; similarly $\mathrm{c}=2$ for $\operatorname{rtPS}$ traffic, $\mathrm{c}=3$ for nrtPS traffic and $\mathrm{c}=4$ for $\mathrm{BE}$ traffic. The service time (transmission time) has an exponential distribution with service rates $\mu_{1}, \mu_{2}, \mu_{3}$ and $\mu_{4}$. Total average service capacity of the system is's' packets/sec. At any instant of time either a packet is appended or departed from any of the four queues. 
The state space diagram of the Markov chain for proposed hybrid scheduler for a particular case of queue size $=1$ packet, for each queue, is shown in Fig. (2). State (i, j, k, l) represents the number of i, j, k, 1 packets of classes ertPS, rtPS, nrtPS and BE in their individual queues. A packet appended to queue $\mathrm{Q}_{1}$ changes the system state from $(i, j, k, l)$ to $(i+1, j, k, l)$. Servicing a packet from queue $Q_{1}$ changes the condition from (i, $j, k$, 1) to $(\mathrm{i}-1, \mathrm{j}, \mathrm{k}, \mathrm{l})$. Similarly, the state of each queue changes depending on whether a packet is appended to a queue or departed from it [8]. In Fig. (2), the FTP traffic with an arrival rate $\lambda_{3}$ packets/sec is split in two rates $\lambda_{31}$ and $\lambda_{32}$ by the splitter. The first portion of split FTP traffic has service rate $\mu_{31}$ packets/sec, scheduled by WFQ scheduler and the second portion of split FTP traffic has service rate $\mu_{32}$ packets/sec, scheduled by RR scheduler.

The limiting state probability of the system is resolved using matrix multiplication method. The matrix ' $\pi$ ' stands for the steady state probability row vector and fulfils equations (1) and (2) [8].

$$
\begin{aligned}
& \pi \mathrm{Q}=0 \\
& \pi \mathrm{e}=1
\end{aligned}
$$

where $\mathrm{e}=(1,1,1 \ldots \ldots 1)^{\mathrm{T}}$ is a unit column vector, $\mathrm{Q}$ is a generator matrix. Continuous Time Markov Chain (CTMC) can be analyzed by the generator matrix. The (x,y) element of generator matrix (transition matrix) is given by equation (3).

$$
g_{x y}=\lambda_{x} p_{x y} \quad \text { if } \quad i \neq j=-\lambda_{x} \quad \text { if } \quad i=\mathrm{j}
$$

where $\lambda_{x}$ is the transition rate out of state $x, p_{x y}$ is the probability of going from state $x$ to $y, g_{x y}=\lambda_{x} p_{x y}$ is the transition rate from state $\mathrm{x}$ to $\mathrm{y}[17]$.

Equation (1) signifies that in case of birth and death process, at steady state, the probability of leaving a state is equal to the probability of entering into that state. Equation (2) signifies that the total sum of steady state probabilities is 1 . The steady state probabilities are solved from the balance equations which are resolved from these matrices.

Following balance equations (4) are derived for proposed hybrid scheduler for queues of size = 1 packet:

$$
\begin{gathered}
-\left(\lambda_{1}+\lambda_{2+} \lambda_{3}+\lambda_{4}\right) \pi_{0}+\mu_{1} \pi_{1}+\mu_{2} \pi_{2}+\mu_{3} \pi_{3}+\mu_{4} \pi_{4}=0 \\
\lambda_{1} \pi_{0}-\left(\lambda_{2}+\lambda_{3}+\lambda_{4}+s\right) \pi_{1}=0 \\
\lambda_{2} \pi_{0}-\left(\lambda_{3}+\lambda_{1}+\lambda_{4}+s\right) \pi_{2}+\mu_{1} \pi_{5}+\mu_{31} \pi_{9}=0 \\
\lambda_{3} \pi_{0}-\left(\lambda_{4}+\lambda_{1}+\lambda_{2}+s\right) \pi_{3}+\mu_{1} \pi_{6}+\mu_{4} \pi_{8}+\mu_{2} \pi_{9}=0 \\
\lambda_{4} \pi_{0}-\left(\lambda_{1}+\lambda_{2}+\lambda_{3}+s\right) \pi_{4}+\mu_{1} \pi_{7}+\mu_{32} \pi_{8}+\mu_{2} \pi_{10}=0 \\
\lambda_{2} \pi_{1}+\lambda_{1} \pi_{2}-\left(\lambda_{3}+\lambda_{4}+\mu_{1}\right) \pi_{5}=0 \\
\lambda_{3} \pi_{1}+\lambda_{1} \pi_{3}-\left(\lambda_{2}+\lambda_{4}+\mu_{1}\right) \pi_{6}=0 \\
\lambda_{4} \pi_{1}+\lambda_{1} \pi_{4}-\left(\lambda_{2}+\lambda_{3}+\mu_{1}\right) \pi_{7}=0 \\
\lambda_{4} \pi_{3}+\lambda_{4} \pi_{4}-\left(\lambda_{1}+\lambda_{2}+\mu_{4}+\mu_{32}\right) \pi_{8}+\mu_{1} \pi_{13} \mu_{2} \pi_{14}=0 \\
\lambda_{3} \pi_{2}+\lambda_{2} \pi_{3}-\left(\lambda_{1}+\lambda_{4}+\mu_{2}+\mu_{31}\right) \pi_{9}+\mu_{1} \pi_{11}=0 \\
\lambda_{4} \pi_{2}+\lambda_{2} \pi_{4}-\left(\lambda_{1}+\lambda_{3}+\mu_{2}\right) \pi_{10}+\mu_{1} \pi_{12}+\mu_{3} \pi_{14}=0 \\
\lambda_{3} \pi_{5}+\lambda_{2} \pi_{6}+\lambda_{1} \pi_{9}-\left(\lambda_{4}+\mu_{1}\right) \pi_{11}=0 \\
\lambda_{4} \pi_{5}+\lambda_{2} \pi_{7}+\lambda_{1} \pi_{10}-\left(\lambda_{3}+\mu_{1}\right) \pi_{12}=0 \\
\lambda_{4} \pi_{6}+\lambda_{3} \pi_{7}+\lambda_{1} \pi_{8}-\left(\mu_{1}+\lambda_{3}\right) \pi_{13}= \\
\lambda_{2} \pi_{8}+\lambda_{4} \pi_{9}+\lambda_{3} \pi_{10}+\mu_{1} \pi_{15}-\left(\mu_{2}+\mu_{31}+\lambda_{1}\right) \pi_{14}=0 \\
\lambda_{4} \pi_{11}+\lambda_{3} \pi_{12}+\lambda_{2} \pi_{13}+\lambda_{1} \pi_{14}-\mu_{1} \pi_{15}=0
\end{gathered}
$$




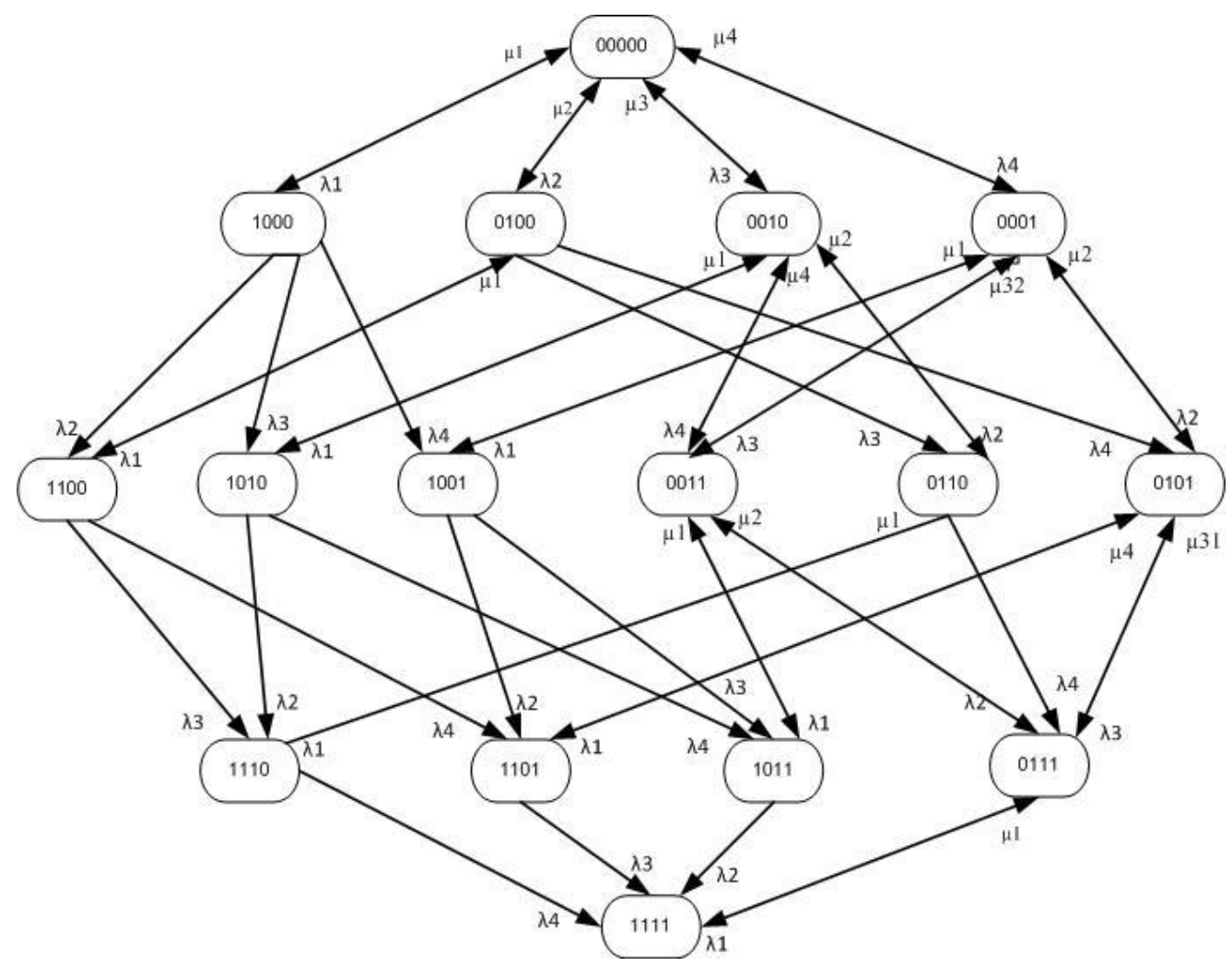

Fig.2. Proposed hybrid scheduler with split traffic's state space diagram for queues of size $=1$ packet

The steady state probability $p_{m}^{c}$ of getting m number of packets of class $c(c=1,2,3,4)$ in the respective buffer can be obtained from joint state probability $\mathrm{p}_{\mathrm{ijkl}}$ using equations (5) to (8).

$$
\begin{aligned}
& p_{m}^{c}=\sum_{l=0}^{K_{4}} \sum_{k=0}^{K_{3}} \sum_{j=0}^{K_{2}} p_{m j k l} \text { for class } 1 \\
& p_{m}^{c}=\sum_{l=0}^{K_{4}} \sum_{k=0}^{K_{3}} \sum_{i=0}^{K_{1}} p_{i m k l} \text { for class } 2 \\
& p_{m}^{c}=\sum_{l=0}^{K_{4}} \sum_{j=0}^{K_{2}} \sum_{i=0}^{K_{1}} p_{i j m l} \text { for class } 3 \\
& p_{m}^{c}=\sum_{k=0}^{K_{3}} \sum_{j=0}^{K_{2}} \sum_{i=0}^{K_{1}} p_{i j k m} \text { for class } 4
\end{aligned}
$$

Performance metrics can be obtained using the probabilities $p_{m}^{c} \quad$ for each class (using equations (9) to (12)). If $\mathrm{K}_{\mathrm{c}}$ is the buffer size for traffic of class $\mathrm{c}$, the mean number of packets of a traffic ' $c$ ' is $\mathrm{L}^{\mathrm{C}}$ (given by (9)).

$$
L^{C}=\sum_{m=0}^{K_{C}}\left[m * p_{m}^{c}\right]
$$

Similarly, the throughput $\mathrm{T}^{\mathrm{C}}$ (explained as a mean rate at which packets of class $\mathrm{c}$ are put in transmit buffer by a scheduler at steady state) is given by (10). 


$$
T^{C}=\lambda_{c} * \sum_{m=0}^{K_{c}-1}\left[p_{m}^{c}\right]
$$

Mean queuing delay for packets of class c traffic $\left(\mathrm{D}^{\mathrm{C})}\right.$ is given by (11).

$$
D^{C}=\frac{L^{c}}{T^{c}}
$$

Fairness index (F) of the scheduler is given by (12) [18].

$$
F=\frac{\left(\sum_{i=1}^{4} T^{i}\right)^{2}}{4 * \sum_{i=1}^{4}\left(T^{i}\right)^{2}}
$$

\section{Performance Analysis of Proposed Hybrid Scheduler for Various Queue Sizes}

This section deals with the investigations of the impact of weight ratios of video and FTP traffic flows scheduled by WFQ on performance metrics such as throughput, mean queuing delay, mean number of packets in a queue of each traffic class, and inter-class fairness using numerical simulation techniques. The size of the buffer queues considered for various configurations are : (i) $K_{1}=K_{2}=K_{3}=K_{4}=1$ packet (ii) $K_{1}=1, K_{2}=4, K_{3}=3$, $\mathrm{K}_{4}=5$ packets, and (iii) $\mathrm{K}_{1}=1, \mathrm{~K}_{2}=3, \mathrm{~K}_{3}=5, \mathrm{~K}_{4}=7$ packets. As voice traffic is usually given the highest priority, a large queue size to buffer voice packets is generally not required, therefore, $\mathrm{K}_{1}=1$ is taken for analysis for all three cases. The maximum queue sizes are varied in random fashion to prove the validity of the considered scheduler in satisfying the objectives of a scheduler for any configuration of queue buffer size. Presently, in Internet traffic, the proportion of voice and video traffic is $10 \%$ and the proportion of FTP and data traffic is $90 \%$. The proportion of voice and video is likely to grow in the near future [19]. Looking at this scenario of Internet traffic, following values have been assigned for arrival rate of traffic flows: for voice traffic $\lambda_{1}=1$ packet $/ \mathrm{sec}$, for video traffic $\lambda_{2}=2$ packets $/ \mathrm{sec}$, for FTP traffic $\lambda_{3}=3$ packets $/ \mathrm{sec}$, for data traffic $\lambda_{4}=4$ packets/sec. As there is no packet drop, the service rate $(\mathrm{s}=10$ packets $/ \mathrm{sec})$ of the model is considered to be equal to the aggregation of all four arrivals.

In Fig. (3), the variations of mean number of packets of voice, video, FTP and data (buffer queue size of $K_{1}=$ $\mathrm{K}_{2}=\mathrm{K}_{3}=\mathrm{K}_{4}=1$ ), shows that the voice traffic has constant mean queue length of packets as voice traffic is given highest priority and is unaffected by weights. At lower weight, lesser number of video packets are serviced causing higher mean number of packets and vice versa. The video and FTP traffic display opposite behaviour, as the weight assigned to video traffic increases, the weight of FTP traffic decreases. The data traffic with lowest priority has highest mean queue length of packets. Thus, the proposed scheduler provides lowest mean queue length of packets for voice traffic which is the major design goal of a scheduler.

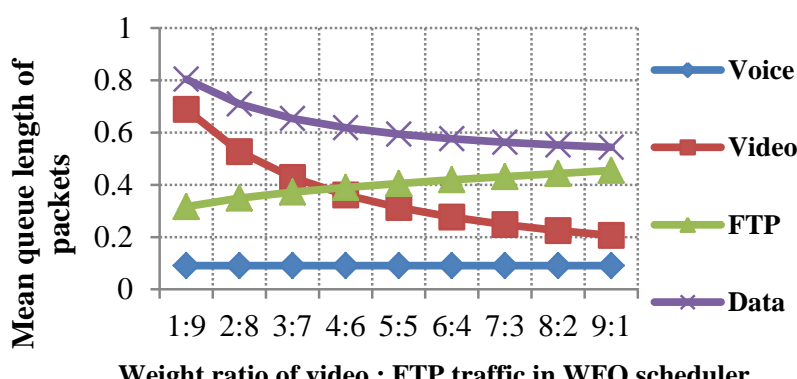

Weight ratio of video : FTP traffic in WFQ scheduler

Fig.3.Mean queue length of packets of triple-play services for $K_{1}=K_{2}=K_{3}=K_{4}=1$ 
Fig. (4) and Fig. (5) show the variations of mean queuing delay of voice, video, FTP and data traffic with variations in weight ratio of WFQ scheduler. As mean queue length of packets of voice traffic is lowest, the voice traffic incurs lowest and constant mean queuing delay. The mean queuing delay of video and FTP traffic reduces when weight ratio increments as departure of packets is in proportion to their weights. Data traffic has highest mean queuing delay as it has lowest priority and highest mean number of packets in the buffer. Thus, the proposed scheduler satisfies the requirement of lower delay for voice and video traffic. The mean queuing delay of video traffic can be obtained at lower weight ratio. Requirement of a lower delay is not a mandatory QoS requirement for data and FTP traffic.

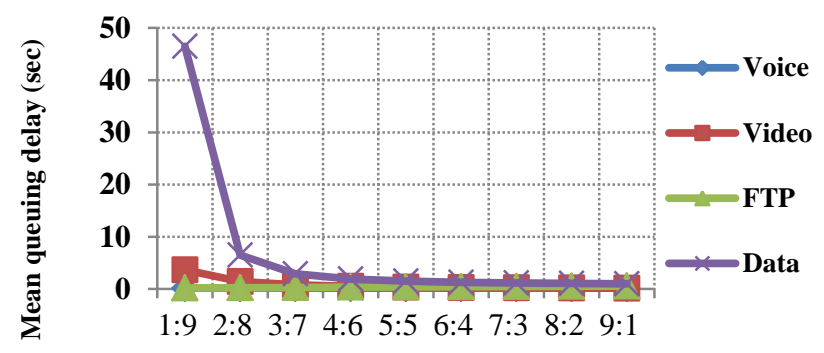

Weight ratio of video : FTP traffic in WFQ scheduler

Fig.4. Mean queuing delay of triple-play services for $\mathrm{K}_{1}=1, \mathrm{~K}_{2}=4, \mathrm{~K}_{3}=3, \mathrm{~K}_{4}=5$

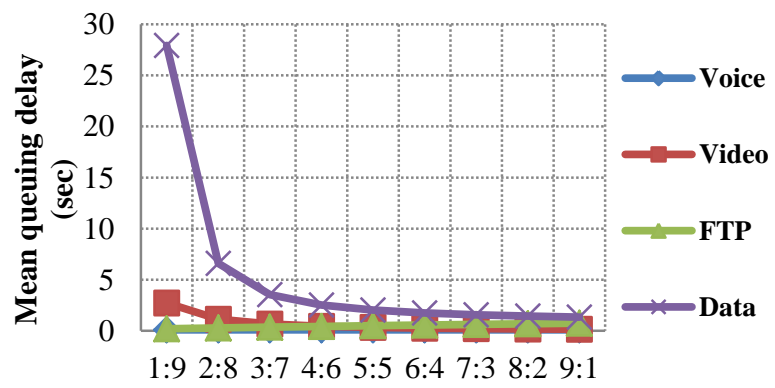

Weight ratio of video : FTP traffic in WFQ

Fig.5. Mean queuing delay of triple-play services for $\mathrm{K}_{1}=1, \mathrm{~K}_{2}=3, \mathrm{~K}_{3}=5, \mathrm{~K}_{4}=7$

Fig. (6) and Fig. (7) show the variations of throughput of voice, video, FTP and data traffic with variations in WFQ scheduler's weight ratio. The voice traffic throughput is lowest and constant as voice traffic has lowest buffer queue size and has lowest arrival rate among all traffics. The throughput of video traffic and first split portion of FTP traffic, scheduled by WFQ scheduler, increases with the increase in weight ratio. The FTP traffic has higher traffic arrival rate, therefore, it has higher throughput than video traffic. Though, data traffic has higher arrival rate than FTP traffic, data packets are scheduled only in absence of voice or video packets in the queue. Hence, the throughput of data packets is lower than that of FTP traffic. Thus, the QoS requirement of high throughput for FTP and data traffic is satisfied by the proposed scheduler and the FTP traffic is not starved under the presence of high priority real-time traffic. 


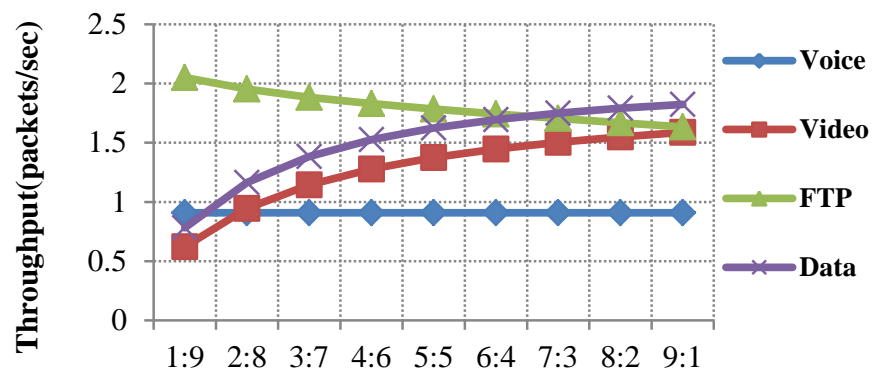

Weight ratio of video : FTP traffic in WFQ scheduler

Fig.6. Throughput of triple-play services for $\mathrm{K}_{1}=\mathrm{K}_{2}=\mathrm{K}_{3}=\mathrm{K}_{4}=1$

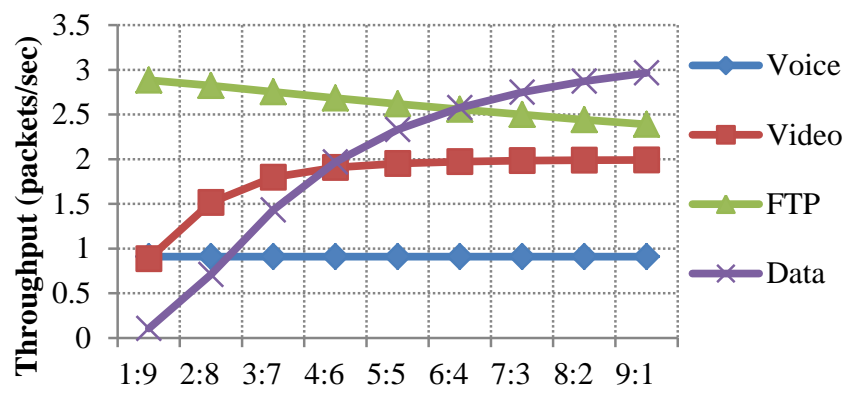

Weight ratio of video : FTP traffic in WFQ scheduler

Fig.7. Throughput of triple-play services for $K_{1}=1, K_{2}=4, K_{3}=3, K_{4}=5$

Fig. (8), Fig.9 and Fig.10 show the variations of inter-class fairness with variations in WFQ scheduler's weight ratio. Unfairness arises when lower priority traffic are starved from scheduling under the presence of large amount of higher priority traffic. The proposed scheduler includes the splitting of FTP traffic that has same priority as that of video traffic in serving the packets. As a result, FTP traffic is not starved of servicing. For a higher weight ratio (e.g. 9:1), as the second split portion of FTP traffic increases, throughput of data traffic increases. Therefore, the inter-class fairness, which depends on throughput of all applications, improves for higher weight ratios of video: FTP traffic. The inter-class fairness is lower for lower weight ratios of WFQ scheduler. Thus, the proposed scheduler on an average provides a high inter-class fairness of about 0.83 for all major weight ratios.

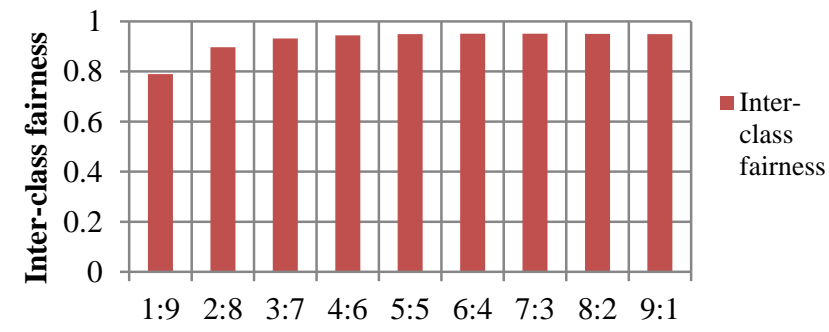

Weight ratio of video : FTP traffic in WFQ scheduler

Fig.8. Inter-class fairness for triple-play services for $\mathrm{K}_{1}=\mathrm{K}_{2}=\mathrm{K}_{3}=\mathrm{K}_{4}=1$ 


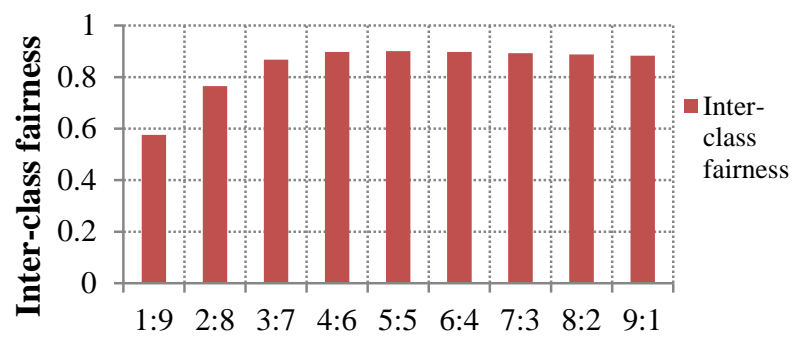

Weight ratio of video : FTP traffic in WFQ scheduler

Fig.9. Inter-class fairness for triple-play services for $K_{1}=1, K_{2}=4, K_{3}=3, K_{4}=5$

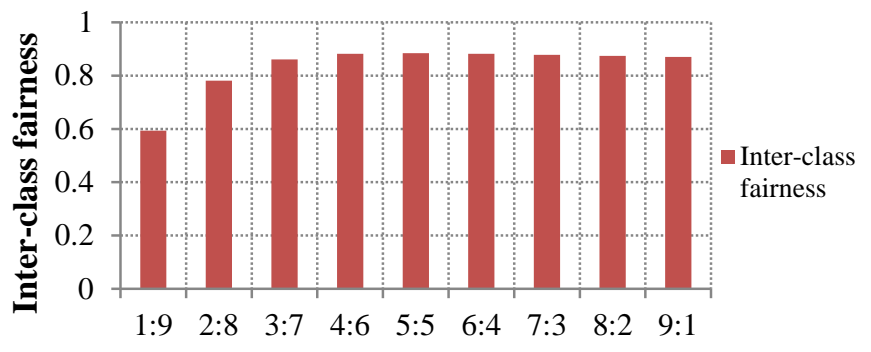

Weight ratio of video : FTP traffic in WFQ scheduler

Fig.10. Inter-class fairness for triple-play services for $\mathrm{K}_{1}=1, \mathrm{~K}_{2}=3, \mathrm{~K}_{3}=5, \mathrm{~K}_{4}=7$

Fig.(11) compares the mean queuing delay of voice traffic for scheduler proposed in this paper with hybrid schedulers proposed in [8] and [9] for different weight ratios of WFQ scheduler. It is observed that the proposed Hybrid Scheduler with Split Traffic (called as HS-ST or model 2) and Wang's model proposed in [8] provide lower queuing delay for voice traffic which is independent of WFQ scheduler's weight ratio. The performance in both the cases is so close that the lines in the graphs overlap each other.This happens because voice traffic is given the highest priority in both models, thereby providing lower queuing delay for voice traffic. The model proposed in [9] is named as Improved Hybrid Scheduler model (IHS model or model 1). In IHS model voice traffic is scheduled through WFQ scheduler, therefore, the mean queuing delay for voice traffic decreas or increases with weight ratio. Thus, IHS model is not suitable for scheduling of voice traffic, particularly, when the overall fraction of voice traffic is higher than other traffics.

Fig. (12) compares the throughput of FTP traffic for scheduler proposed in this paper with hybrid schedulers proposed in [8] and [9] for different weight ratios of WFQ scheduler. It is observed that the proposed HS-ST model and Wang's model provide higher throughput for FTP traffic as compared to IHS model. The first portion of split FTP traffic having high priority has higher throughput as compared to the second split portion of FTP traffic. Moreover, in Wang's model, as FTP traffic is scheduled through WFQ scheduler, its throughput increases with the increase in weight ratio. In IHS model, as FTP traffic has a lower priority than video traffic, throughput performance of FTP traffic degrades. The Wang's model provides highest throughput for FTP traffic as the available resources are divided among three applications only, while in other two models the available resources are divided among four applications. Hence, it can be concluded that proposed HS-ST model is most suitable one for FTP traffic as it satisfies the high throughput requirement of FTP traffic along with satisfying QoS requirements of voice, video and data traffic. 


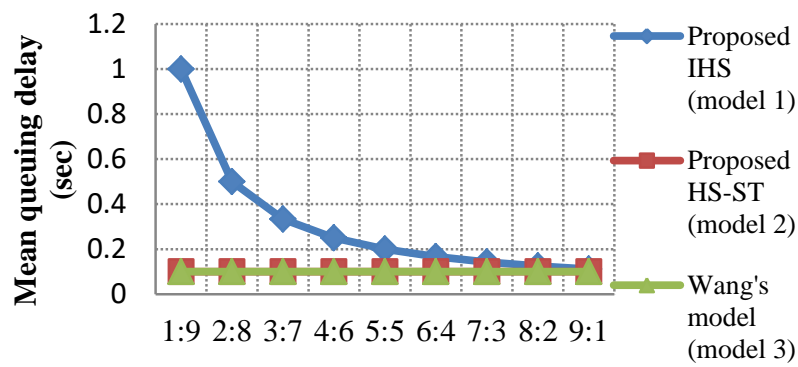

Weight ratio of WFQ scheduler

Fig.11. Comparison of mean queuing delay of voice traffic for proposed hybrid scheduler for $\mathrm{K}_{1}=1, \mathrm{~K}_{2}=3, \mathrm{~K}_{3}=5, \mathrm{~K}_{4}=7$

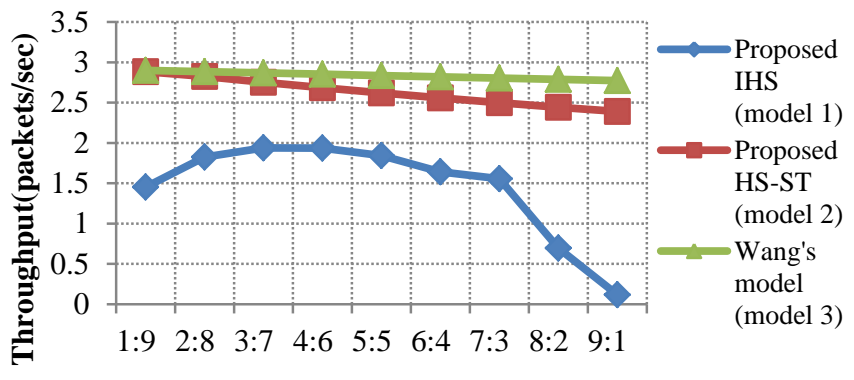

Weight ratio of WFQ scheduler

Fig.12. Comparison of throughput of FTP traffic for proposed hybrid schedulers for $\mathrm{K} 1=1, \mathrm{~K} 2=4, \mathrm{~K} 3=3$, $\mathrm{K} 4=5$

Fig. 13 compares the inter-class fairness of hybrid scheduler proposed in this paper with hybrid schedulers proposed in [8] and [9] for different weight ratios of WFQ scheduler. As compared to Wang's model, IHS model and the HS-ST model, on an average, provide high inter-class fairness. The IHS model (model 1) provides the highest value of inter-class fairness for most of the weight ratios as there are only two levels of priority for scheduling of the four classes of traffic and, unlike models 2 and 3, not a single traffic class is given the highest priority. Hence, the allocation of resources among different traffic classes is fair. HS-ST model (model 2) provides moderate fairness as compared to IHS model (model 1) as the scheduling of voice traffic with highest priority poses unfairness in servicing packets of the rest of the three traffic classes.

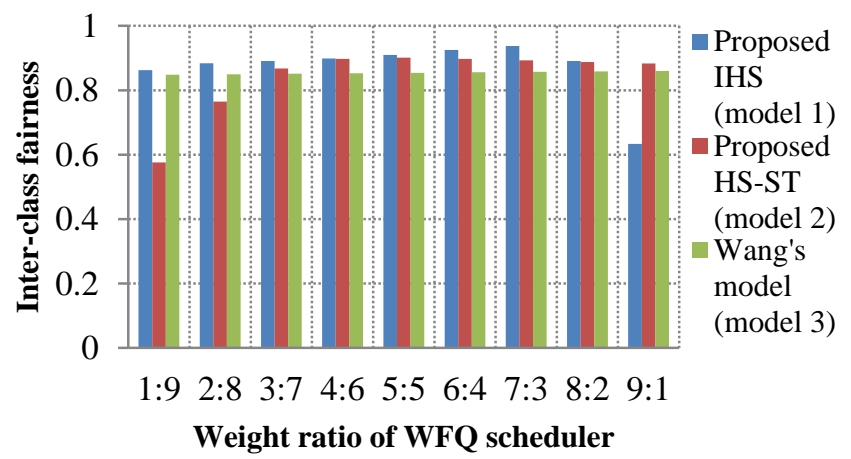

Fig.13. Comparison of inter-class fairness for proposed hybrid schedulers for $K_{1}=1, K_{2}=4, K_{3}=3, K_{4}=5$ 
For voice traffic, the proposed HS-ST model provides mean queuing delay lowered by $68.18 \%$ as compared to IHS model at a particular weight ratio. Major advantage of HS-ST model over IHS model is an increase in throughput of FTP traffic by $67.27 \%$ which is the necessary parameter for QoS requirement of FTP traffic. Both the models offer same throughput for data traffic. The IHS model on an average provides an inter-class fairness of 0.89 while HS-ST model provides an inter-class fairness of 0.83 . It is observed that the proposed HS-ST model satisfies the requirement of lower mean queuing delay of voice traffic and higher throughput of FTP traffic along with scheduling of data traffic and provides moderate inter-class fairness. Therefore, the model proposed in this paper is most suitable for a large variety of heterogeneous traffic applications. The Wang's model does not have separate scheduling of data traffic and, therefore, it is not suitable for heterogeneous traffic such as voice, video, FTP and data.

\section{Conclusions}

With the increase in demand for multimedia applications in wireless networks, an IEEE 802.16 based WiMAX networks represent a viable integrated solution for scheduling of heterogeneous traffic for satisfying their various QoS requirements. A hybrid scheduler with split FTP traffic has been proposed in this paper to satisfy QoS demands of triple-play services. An analytical model is developed using queuing theory and Markov chain techniques to evaluate the performance metrics of the proposed model such as mean queue length of packets, mean queuing delay and throughput as a function of weight ratios of WFQ scheduler and buffer sizes. The analysis is used to further investigate the effectiveness of the proposed hybrid scheduler in satisfying design goals such as the QoS requirements of each application, inter-class traffic fairness etc. using simulation techniques.

The proposed HS-ST model (model 2) satisfies the requirements of lower mean queuing delay for voice and video traffic, as well as high throughput requirement of FTP and data traffic, thus fulfilling the QoS requirements of all four types of applications. The inclusion of a splitter in this model improves throughput of FTP traffic and provides high inter-class fairness. Further, the proposed HS-ST model is also compared with Improved Hybrid Scheduler (IHS) model and Wang's model. The proposed HS-ST model provides lower queuing delay for voice traffic (lowered by $68.18 \%$ ) and a higher throughput for FTP traffic (increased by $67.27 \%$ ) as compared to IHS model. Therefore, the proposed hybrid scheduler with split traffic is most suitable for a large variety of heterogeneous traffic applications as compared to IHS model and Wang's model. The time varying nature of wireless channel poses a challenge in QoS provisioning [20]. Including the impact of time varying nature of wireless channel on the performance of proposed scheduler to optimize the throughput of various traffics along with satisfying the QoS constraints (such as delay) can be the topic of further research.

\section{References}

[1] So-In C, Jain R, and Tamimi A K. "Scheduling in IEEE 802.16e Mobile WiMAX Networks: Key Issues and a Survey", IEEE Journal On selected Areas in Communications 2009; 27(2): 56-171.

[2] Nuaymi L (2007) Wi-MAX Technology for Broadband Wireless Access. John Wiley and Sons Ltd.

[3] Yadav A, Vyavahare P D, and Bansod P P. "Review of WiMAX Scheduling Algorithms and their Classification, Journal of Institution of Engineers", India, series B (Springer) 2015; 96(2): 197-208.

[4] Shrivastava A, Bansod P, Gupta K, and Merchant S N. "An improved multicast based energy efficient opportunistic data scheduling algorithm for VANET", AEU-International Journal of Electronics and Communications 2018; 83 : 407-415.

[5] Andrews Jeffrey G., Ghosh A. and Muhamed R. (2007) Fundamentals of WiMAX: Understanding Broadband Wireless Networking, $1^{\text {st }}$ ed., Prentice Hall, USA. 
[6] Taghipoor M, MJafari S. and Hosseini V. (2012) Scheduling Algorithm and Bandwidth Allocation in WiMAX. In Quality of Service and Resource Allocation in WiMAX. Open access peer-reviewed chapter edited by Dr. Roberto Hincapie, InTech.

[7] Nandhini R, and Devarajan N. "Comparison for WiMAX Scheduling Algorithms and Proposal Quality Of Service Improvement in WiMAX Network", American Journal of Applied Sciences 2014; 11(1): 8-16.

[8] Wang L, Min G, Kouvatsos D D, and Jin X. "Analytical Modeling of an Integrated Priority and WFQ Scheduling scheme in Multi Service Networks", Journal of Computer communications 2010; 33(11): 93-101.

[9] Yadav A, Vyavahare P D, and Bansod P P. "An Improved Hybrid Scheduler for WiMAX and its Performance Evaluation". International Conference on Networking and Service (ICNS), Chamonix, France, April 2014: 84- 89.

[10] Jin X, and Min G. "Modeling and analysis of priority queuing systems with multi-class self-similar network traffic: a novel and efficient queue-decomposition approach", IEEE Transactions on Communications 2009; 57 (5):1444-1452.

[11] Noordin K A, and Markarian G. "Providing QoS Support through Scheduling in WiMAX Systems". International Journal of the Physical Sciences 2011; 6 (16): 4070-4081.

[12] Kafhali S El, and Hanini M. "Stochastic Modeling and Analysis of Feedback Control on the QoS VoIP Traffic in a single cell IEEE 802.16e Networks". International Journal of Computer Science 2017; 44(1): 19-28.

[13] Ahmed Z, and Hamma S. "Analytical model of two level scheduling algorithm for WiMAX networks". International Conference on Wireless Networks and Mobile Communications (WINCOM), Marrakech, Morocco, Oct 2015.

[14] Grover P, and Teixeira A M. "A New Queue Length based Scheduling Strategy for nrtPS Service Class in IEEE 802.16 Networks". 13 ${ }^{\text {th }}$ International Conference on Wireless Networks and Mobile Systems (WINSYS), Lisbon, Portugal, July 2016.

[15] Sreenivasulu U., Naganjaneyulu P.V., and Giriprasad M.N. "Downlink Scheduling Algorithm for WiMAX Protocol to Improve QoS". Arab Journal of Science and Engineering, 2017 https://doi.org/10.1007/s13369-017-2717-6.

[16] Dhrona P, Abu Ali Najah, and Hassanein H. "A Performance study of Uplink scheduling Algorithms in point to Multipoint WiMAX Networks", Journal of Computer Communication 2009; 32(3): 511-52.

[17] Hossein P N. Introduction to Probability, Statistics, and Random Processes. Kappa Research, 2014.

[18] Jain R, Chiu D M, and Hawe W. "A Quantitative Measure of Fairness and Discrimination for Resource Allocation in Shared Computer Systems". DEC Research Report TR-301 1984.

[19] Aejandro Q, Yacine E, and Samuel P. "Performance evaluation of a broadband wireless access system subjected to heavy load". Journal of Computer Communication 2004; 27: 781-79.

[20] Karandikar A, and Salodkar N. "Cross Layer Scheduling in Wireless Networks", in: Kennington Jeff, Olinick El, Rajan Dinesh (Eds.), Wireless Network Design: Optimization Models and Solutions Procedures, Springer- Verlag 2010.

\section{Authors' Profiles}

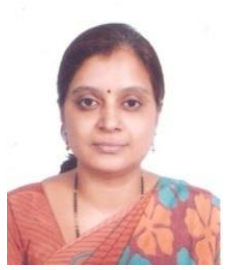

Mrs. Anju Lata Yadav obtained B.E. degree in Electronics from Ujjain Engg. College, Ujjain,(MP) in 1996 and M.E. degree in Electrical Engineering from Shri G.S. Institute Of Technology and Sc. Indore(MP) in 2002. Her major field of study is Electronics and Communication. She is presently an Associate Professor in Shri G.S. Institute Of Technology and Sc. Indore (MP), India. Mrs. Yadav is a member of IETE, ISTE and IE. She has published papers in International Journal, National and International Conferences. Her research interests are QoS in WiMAX, Schedulers in WiMAX and QoS in MPLS. 


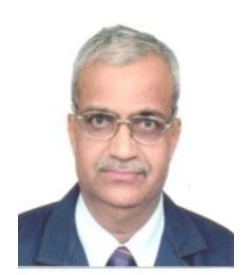

Italy since 1998 .

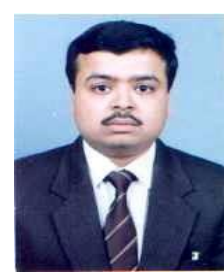

Dr. Prashant P. Bansod received B.E. degree in Electronics and Instrumentation Engineering from Shri G.S. Institute Of Technology and Sc., Indore (MP) in 1989, M.Tech in Electronics Engineering from VNIT, Nagpur in 1992 and Ph.D. in Biosciences and Bioengineering from IIT Bombay in 2010. His fields of specialization include Medical Signal, Image processing, and VLSI Design. He is presently an Associate Professor and Head of the Department in Shri G.S. Institute Of Technology and Sc. Indore (MP), India. He has more than 22 research articles to his credit. He is a member of ISTE, IE and Instrument Society of

Dr.Prakash D. Vyavahare obtained his M.Tech and Ph.D. degrees from IIT Bombay in 1976 and 1994 respectively. His major field of study is Electronics and Communication. He is presently Professor and Head of the Department in Shri G.S. Institute Of Technology and Sc. Indore (MP), India. He has more than 40 research articles to his credit. His research interests are characterization of Mobile Radio Channel, Computer Networking and Channel Coding Techniques. He is a senior member of IEEE, Fellow member of IETE and IE. He was Hindu Hitachi scholar during 1981-82 and an Associate member of ICTP-UNESCO,

India.

How to cite this paper: Anju Lata Yadav, P. D. Vyavahare, P. P. Bansod, "Proposed WiMAX Hybrid Scheduler with Split FTP Traffic and its Performance Evaluation", International Journal of Wireless and Microwave Technologies(IJWMT), Vol.8, No.6, pp. 1-14, 2018.DOI: 10.5815/ijwmt.2018.06.01 